\title{
Envy-free cake divisions cannot be found by finite protocols
}

\author{
Walter Stromquist* \\ Department of Mathematics and Statistics \\ Swarthmore College, Swarthmore, PA 19081 \\ wstromq1@swarthmore.edu
}

Submitted: Oct 21, 2007; Accepted: Dec 23, 2007; Published: Jan 7, 2008

Mathematics Subject Classifications: 91B32, 68Q17, 05D05

\begin{abstract}
We show that no finite protocol (even if unbounded) can guarantee an envy-free division of a cake among three or more players, if each player is to receive a single connected piece.
\end{abstract}

\section{Cutting cakes}

An early result for cake cutting [5] is that a cake can always be divided among $n$ players, using $n-1$ cuts, in such a way that no player considers another player's piece to be more valuable than its own. In this paper we show that no finite protocol can guarantee finding such a division.

As usual we represent the cake as the unit interval $[0,1]$. Possible cuts are points in the interval and possible pieces are subintervals $S=[x, y] \subseteq[0,1]$. The limitation to $n-1$ cuts means that each player's piece consists of a single connected interval. Players' preferences are represented by nonatomic measures on $[0,1]$. We write $v_{i}$ for the $i$-th player's measure, and either $v_{i}(S)$ or the shorthand $v_{i}(x, y)$ for the value to the $i$-th player of the piece $S=[x, y]$. The requirement that the measures be nonatomic implies that $v_{i}(x, x)$ is always zero. (It also allows us to represent pieces as closed intervals without worrying about the values of the endpoints.) Note that the measures are determined by the values of $F_{i}(x)=v_{i}(0, x)$ for all $i$ and $x$. We assume throughout that $v_{i}(0,1)=1$, so that each $v_{i}$ is a probability measure and each $F_{i}$ is actually a (cumulative) distribution function.

${ }^{*}$ This paper arose from discussions at the Dagstuhl Seminar on Fair Division, June, 2007. The author thanks the organizers of the seminar and all those who make the Dagstuhl program possible. 
For the measures we consider in this paper, $v_{i}(x, y)$ is always positive when $0 \leq x<$ $y \leq 1$. This implies that the measures are absolutely continuous with respect to each other, and also that the functions $F_{i}$ are bijections on $[0,1]$. (This restriction does not affect the generality of our main result. If finite protocols cannot handle a restricted class of measures, then they cannot handle a more general class.)

An allocation of pieces to players, piece $S_{i}$ being assigned to player $i$, is called envy free if $v_{i}\left(S_{i}\right) \geq v_{i}\left(S_{j}\right)$ for all $i$ and $j$. The result mentioned above is that for any system of players' measures, there is an envy-free division in which each player receives a single interval. One proof of this result is Su's argument in [6], which works for all $n$. Su's method is constructive in the sense that an explicit sequence of divisions is constructed, with the actual envy-free division as its limit. Because it requires convergence, it is not a finite protocol.

Other non-finite procedures are available in the case of $n=3$. A moving-knife algorithm was given in [5], and a simpler moving knife algorithm in [1]. Both of these procedures involve continuous operations. Since each of the procedures requires that players watch more than one knife simultaneously, there is no obvious way of replacing the knives with a finite bidding or trimming process. So these procedures cannot be called finite, either.

By contrast, there is a simple finite protocol for dividing a cake between two people - "I cut, you choose." There is also a finite protocol for envy-free division among three people, if five cuts are allowed and each player receives two of the resulting six pieces (the ConwayGuy-Selfridge procedure; see, for example, [2]). One might ask - and Steven Brams did ask, at the Dagstuhl Fair Division Seminar [3] — whether there is a finite protocol in the case of three players, two cuts, or more generally $n$ players, $n-1$ cuts with $n \geq 3$. The purpose of this paper is to answer this question in the negative.

The first issue, of course, is what constitutes a finite protocol. We address this in Sections 2 and 3, following the paper by Woeginger and Sgall [7]. We do not require that the number of steps be bounded in advance; as a consequence, the conclusion of this paper applies to both bounded and unbounded protocols, as long as they are finite.

In Sections 4 and 5 we prove the main theorem for $n=3$. In Section 4 we introduce a special class of examples which we call "rigid measure systems." A rigid measure system consists of three measures $v_{A}, v_{B}, v_{C}$ with certain properties. (When $n=3$ we index the players by $i=A, B, C$.) A consequence of these properties is that there is a unique pair of cuts that produces an envy-free division among players with these measures. In Section 5 we combine these properties to prove that there is no finite protocol for the case of three players, two cuts, even if we assume that the players' measures form a rigid measure system. The case of $n>3$ is addressed in Section 6 .

One way to understand the proof is to consider the plight of a person who is asked to guess an arbitrary real number. He is told only whether each guess is too high or too low. Clearly there is not much hope for a finite protocol. The case of finding an envy-free division, given a rigid measure system, is similar. One must find exactly the right cuts, and they are as hard to guess as arbitrary real numbers. 


\section{What are finite protocols?}

We recommend the paper of Woeginger and Sgall [7] for its discussion of finite protocols, building on the framework of Robertson and Webb [4]. Like Robertson and Webb, Woeginger and Sgall are concerned with counting steps in their protocols. In this paper we are only concerned with whether the protocols are finite, which means that we can gloss over some issues that Woeginger and Sgall treat more completely.

In this paper, a finite protocol is one in which only finitely many marks are made on the cake. Formally, a mark is a real number $x \in[0,1]$; informally, it is a physical mark on the cake that is visible to all players. A protocol proceeds in steps. In each step, the protocol selects a player $i$ and a real number $\alpha$, and invites player $i$ to make a mark at the unique point $x$ for which $v_{i}(0, x)=\alpha$. (Woeginger and Sgall call $x$ the player's " $\alpha$-point." We assume that the players answer honestly.) After any step, the protocol may terminate by cutting the cake at $n-1$ of the existing marks and allocating the pieces to the players.

We assume that whenever a mark is made at $x$, the players reveal the values of $v_{j}(0, x)$ for all $j$. As a consequence, the values of $v_{j}(x, y)$, for all $j$ and all existing marks $x$ and $y$, are common knowledge. Except for this information, the protocol operates without direct knowledge of the player's measures. The choices of $i$ and $\alpha$ at each step may be determined by the protocol in any manner, provided that they are based only on the existing marks and the knowledge of the players' measures so far revealed. Similarly, the choices of whether to terminate at any step, how to cut the cake, and how to allocate the pieces may be determined in any manner based on the same information.

A protocol is finite if it is guaranteed to terminate after finitely many steps, whatever the players' measures. It is bounded if, for some integer $N$, it is guaranteed to terminate after at most $N$ steps. Our theorem applies to finite protocols, whether bounded or not.

It is worth restating these notions in the framework of Woeginger and Sgall. They define two types of queries:

- Marking query: The protocol selects a player $i$ and a real number $\alpha$; the player marks the cake at the least $x$ for which $v_{i}(0, x)=\alpha$.

- Value query: The protocol selects a player $i$ and an existing mark $x$; the player provides the value of $v_{i}(0, x)$.

(Woeginger and Sgall use the word "cuts" for what we call marks. We prefer to reserve "cut" for a mark that actually becomes the endpoint of some player's piece.)

The protocol is using the players as oracles. Player $i$ can provide single evaluations of $F_{i}(x)$ (value queries) or $F_{i}^{-1}(\alpha)$ (marking queries). A finite protocol is one that consults oracles finitely many times.

In this paper we are saying, in effect, that one marking query equals one step. Effectively we are allowing all possible value queries to be made after each step. Since each new mark enables only finitely many new value queries, this seems like a reasonable convention for the problem at hand. (It is also used by Robertson and Webb.)

It would not change our result (or proof) if we were to allow steps in which the protocol calculates a mark directly from the available information. Nor would it matter if we were 
to allow random protocols - since no random protocol can guarantee a result after finitely many steps unless every realization of it, treated as a deterministic protocol, can make the same guarantee.

\section{$3 \quad$ Examples of protocols}

We give two examples of protocols, one that we accept as finite and one that we reject.

Example: A finite, unbounded protocol that does not work. Consider this protocol: Enumerate the rational numbers, and for each rational number $\alpha$, ask each player to mark the cake at its $\alpha$-point. Terminate whenever an envy-free division is possible using existing marks. (Alternatively, we might just as well enumerate the algebraic numbers. We could also allow the protocol to mark the cake directly at rational or algebraic points.)

A protocol of this type would be finite, and would always find an envy-free allocation, if it were the case that for any set of measures there is an envy-free allocation with rational piece values (or algebraic values, or rational or algebraic piece widths). We will see in the next section that there are systems of measures for which no such envy-free allocations exist. Therefore protocols of this form cannot be guaranteed to terminate.

From here we could head into logical thickets. Given some notion of "constructible" numbers, we could enumerate them and design a protocol as above. It would find any envy-free solution in which the piece widths or piece values are all constructible. To draw the conclusion we want, we must claim that there are measure systems for which no such envy-free allocations exist. Looking ahead to the next section, we are claiming that there are rigid measure systems whose parameters $x, y, s, t$ are not constructible numbers. In effect, the players are allowed to construct their preferences in ways in which the protocol is not allowed to construct numbers. We will go no further in this direction.

Example: A "cheating" protocol. Consider this sequence of events (from Woeginger and Sgall): The first query is addressed to player 1. The player ignores the content of the query. Instead, the player codes its entire measure into a real number $x$, and marks the cake at $x$. Each of players 2 through $n-1$ then does the same thing. The next query is addressed to player $n$, who observes the existing marks, infers the other players' measures in their entirety, and calculates an envy-free allocation off line, perhaps using Su's algorithm. Player $n$ then marks the cake at the point where the first cut should be made. In response to more queries, player $n$ reveals the rest of the cuts, and then the algorithm terminates.

This is a finite algorithm that guarantees an envy-free allocation, but it is utterly foreign to the spirit of cake cutting. We turn away from it in horror.

More practically, we exclude this protocol by requiring that the players answer honestly. As a result, in a finite algorithm, each player is allowed to announce only finitely many of its $\alpha$-points. Woeginger and Sgall address this issue further. Considerations related to this example are the only serious limitation on the kinds of steps we can allow in a finite protocol. 


\section{Rigid Measure Systems}

In this section we define rigid measure systems. Let $x, y, s$, and $t$ satisfy $0<x<y<1$, $0<s<1 / 3<t<1 / 2$, and $s+2 t=1$. Then a rigid measure system (RMS) with parameters $x, y, s, t$ is a set of three measures $\left\{v_{i}\right\}$ for $i=A, B, C$ with these two properties:

1. The density of each measure is always strictly between $(\sqrt{2} / 2)$ and $\sqrt{2}$. Equivalently: If $S$ is a piece of length $\ell$, then

$$
(\sqrt{2} / 2) \ell<v_{i}(S)<\sqrt{2} \ell
$$

for every $i$.

2. The player's values for the pieces defined by $x$ and $y$ are given by the following table.

\begin{tabular}{c|ccc} 
& {$[0, x]$} & {$[x, y]$} & {$[y, 1]$} \\
\hline$v_{A}$ & $t$ & $t$ & $s$ \\
$v_{B}$ & $s$ & $t$ & $t$ \\
$v_{C}$ & $t$ & $s$ & $t$
\end{tabular}

We need some lemmas about rigid measure systems. The first lemma says that players in an RMS cannot differ too greatly in their comparisons of two pieces.

Lemma 1 Let $i$ and $j$ be two players with measures in a rigid measure system, and let $S_{1}$ and $S_{2}$ be any two pieces. If player $i$ values $S_{1}$ as at least twice the value of $S_{2}$, then player $j$ does not value $S_{2}$ as at least twice the value of $S_{1}$. That is: If

$$
v_{i}\left(S_{1}\right) \geq 2 v_{i}\left(S_{2}\right)
$$

then

$$
2 v_{j}\left(S_{1}\right)>v_{j}\left(S_{2}\right) \text {. }
$$

Proof: This is a consequence of the bounds on the densities of the measures. Suppose $S_{1}$ and $S_{2}$ have lengths $\ell_{1}$ and $\ell_{2}$ respectively, and $v_{i}\left(S_{1}\right) \geq 2 v_{i}\left(S_{2}\right)$. Then

$$
\begin{aligned}
2 v_{j}\left(S_{1}\right) & >2(\sqrt{2} / 2) \ell_{1} \\
& =\sqrt{2} \ell_{1} \\
& >v_{i}\left(S_{1}\right) \\
& \geq 2 v_{i}\left(S_{2}\right) \\
& >2(\sqrt{2} / 2) \ell_{2} \\
& =\sqrt{2} \ell_{2} \\
& >v_{j}\left(S_{2}\right) .
\end{aligned}
$$

The next lemma is the reason for calling these systems "rigid." 
Lemma 2 If $v_{A}, v_{B}$, and $v_{C}$ form a rigid measure system with parameters $x, y, s, t$, then every envy-free division of the cake among $A, B, C$ has cuts at $x$ and $y$.

Proof: First, cuts at $x$ and $y$ do give an envy-free division - in two different ways. The pieces $[0, x],[x, y]$, and $[y, 1]$ can be given to players $A, B$, and $C$ respectively; or, they can be given to $C, A$, and $B$ respectively. In each case, each player assigns value $t$ to its own piece and no more than $t$ to any other player's piece, so the division is envy free.

We will see that no other cuts can give an envy-free division. Suppose that instead of making cuts at $x$ and $y$, we make them at $\hat{x}$ and $\hat{y}$.

If $\hat{x} \leq x$ and $\hat{y} \geq y$ with strict inequality in at least one case, then both $A$ and $B$ would insist on the middle piece. They would each consider its value to be strictly greater than $t$, and other pieces' values to be $t$ or less.

If $\hat{x} \geq x$ and $\hat{y} \leq y$ with strict inequality in at least one case, then no player would accept the middle piece. Each player would consider some other piece to have value at least $t$, and the middle piece to have value strictly less than $t$.

By symmetry we are left only to consider the possibility that $\hat{x} \geq x$ and $\hat{y} \geq y$ with strict inequality in at least one case. It is clear that both $A$ and $C$ now prefer the leftmost piece $[0, \hat{x}]$ to the rightmost piece $[\hat{y}, 1]$, so if the division is to be envy free the rightmost piece must be accepted by $B$. We will show that in that case, $A$ and $C$ both insist on the leftmost piece.

Let $S_{1}=[x, \hat{x}]$ and $S_{2}=[y, \hat{y}]$ - that is, $S_{1}$ and $S_{2}$ are the slivers that distinguish cuts at $\hat{x}, \hat{y}$ from cuts at $x, y$. If $B$ accepts the rightmost piece, it is because

$$
\begin{aligned}
v_{B}(\hat{y}, 1) & \geq v_{B}(\hat{x}, \hat{y}) \\
v_{B}(y, 1)-v_{B}\left(S_{2}\right) & \geq v_{B}(x, y)+v_{B}\left(S_{2}\right)-v_{B}\left(S_{1}\right) \\
t-v_{B}\left(S_{2}\right) & \geq t+v_{B}\left(S_{2}\right)-v_{B}\left(S_{1}\right) \\
v_{B}\left(S_{1}\right) & \geq 2 v_{B}\left(S_{2}\right) .
\end{aligned}
$$

So $B$ considers $S_{1}$ to be twice as valuable as $S_{2}$, and by the previous lemma the other players must consider $S_{2}$ to be less than twice as valuable as $S_{1}$. It follows that

$$
\begin{aligned}
v_{A}(0, \hat{x}) & =t+v_{A}\left(S_{1}\right) \\
& =t-v_{A}\left(S_{1}\right)+2 v_{A}\left(S_{1}\right) \\
& >t-v_{A}\left(S_{1}\right)+v_{A}\left(S_{2}\right) \\
& =v_{A}(\hat{x}, \hat{y}),
\end{aligned}
$$

so player $A$ insists on the leftmost piece. The same is true of player $C$ by a wider margin. Therefore no player will accept the middle piece, and no division with cuts at $\hat{x}$ and $\hat{y}$ can be envy free.

The next lemma tells us that one player in a rigid measure system cannot determine the parameters of the system from its own measure. Further, this remains true even if the player knows the other players' measures outside of neighborhoods of the likely cuts. 
Lemma 3 Let $v_{A}, v_{B}$, and $v_{C}$ form a rigid measure system with parameters $x, y, s, t$. Let $\eta>0$, let $i$ be one of the players, and let $\delta>0$ be such that $1 / 3+\delta<t<1 / 2-\delta$. Let $\hat{x}$ be sufficiently close to $x$. Then there exists a rigid measure system $\hat{v}_{A}, \hat{v}_{B}, \hat{v}_{C}$ with parameters $\hat{x}, \hat{y}, \hat{s}, \hat{t}$ (for some $\hat{y}, \hat{s}, \hat{t}$ ) such that

- $\hat{v}_{i}=v_{i}$; that is, player $i$ 's new measure agrees with its old measure;

- $\hat{v}_{j}(u, v)=v(u, v)$ for all $j, u, v$ if neither $u$ nor $v$ is within $\eta$ of $x$ or $y$. That is, all of the new measures agree with the old measures outside of the specified neighborhoods of $x$ and $y$;

- $\hat{y} \neq y$; and

- $1 / 3+\delta<\hat{t}<1 / 2-\delta$.

The requirement that $\hat{x}$ be sufficiently close to $x$ means that there is an $\epsilon>0$ such that whenever $|\hat{x}-x|<\epsilon$, the lemma applies. The value of $\epsilon$ may depend on $\eta$ as well as on the measures $v_{A}, v_{B}, v_{C}$.

The first two conclusions of the lemma mean that player $i$ cannot tell whether it is participating in the system $v_{A}, v_{B}, v_{C}$ or the system $\hat{v}_{A}, \hat{v}_{B}, \hat{v}_{C}$. The last two conclusions are technical requirements that will be needed in the next section.

Proof: For clarity we will consider only the case of $i=A$. We therefore fix $\hat{v}_{A}=v_{A}$. (The cases of $i=B$ and $i=C$ are not completely symmetrical to this case, but are very similar.)

Choose $\hat{t}$ near $t$-how near, we will determine as we go along. The first of many constraints on the choice of $\hat{t}$ is that $1 / 3+\delta<\hat{t}<1 / 2-\delta$. Like the remaining constraints, it can be satisfied by choosing $\hat{t}$ sufficiently close to $t$.

Set $\hat{s}=1-2 \hat{t}$ and choose $\hat{x}$ and $\hat{y}$ such that $v_{A}(0, \hat{x})=\hat{t}$ and $v_{A}(0, \hat{y})=2 \hat{t}$. This choice forces $\hat{y} \neq y$. Note that $\hat{x}$ and $\hat{y}$ are both continuous functions of $\hat{t}$.

We need for $\hat{x}$ and $\hat{y}$ to satisfy $|\hat{x}-x|<\eta$ and $|\hat{y}-y|<\eta$. These form the second set of constraints on $\hat{t}$, and they are satisfied if $\hat{t}$ is sufficiently close to $v_{A}(0, x)=t$ and $2 \hat{t}$ is sufficiently close to $v_{A}(0, y)=2 t$.

Assume that we have chosen $\hat{t}$ to satisfy the above constraints. We must now construct the measures $\hat{v}_{B}$ and $\hat{v}_{C}$. We do this by assigning values to $\hat{v}_{B}(0, u)$ and $\hat{v}_{C}(0, u)$ for each $u$; these assignments will determine the measures entirely. We assign:

- $\hat{v}_{B}(0, u)=v_{B}(0, u)$ and $\hat{v}_{C}(0, u)=v_{C}(0, u)$ if $u$ is not within $\eta$ of $x$ or $y$.

- $\hat{v}_{B}(0, \hat{x})=\hat{s}$ and $\hat{v}_{B}(0, \hat{y})=\hat{s}+\hat{t}$.

- $\hat{v}_{C}(0, \hat{x})=\hat{t}$ and $\hat{v}_{C}(0, \hat{y})=\hat{s}+\hat{t}$.

- Finally, we extend $\hat{v}_{B}(0, u)$ and $\hat{v}_{C}(0, u)$ linearly to values of $u$ in the intervals $[x-\eta, \hat{x}],[\hat{x}, x+\eta],[y-\eta, \hat{y}]$, and $[\hat{y}, y+\eta]$. 
It is clear that $\hat{v}_{A}, \hat{v}_{B}, \hat{v}_{C}$ form a rigid measure system provided that they meet the density requirements; that is, provided that the densities of $\hat{v}_{B}$ and $\hat{v}_{C}$ stay strictly between $\sqrt{2} / 2$ and $\sqrt{2}$. If they do, then the system clearly has the required parameters $\hat{x}, \hat{y}, \hat{s}, \hat{t}$ and meets the requirements of the lemma.

The measures meet the density requirement if the slopes of $\hat{v}_{B}(0, u)$ and $\hat{v}_{C}(0, u)$ are between $\sqrt{2} / 2$ and $\sqrt{2}$ on all four of the intervals to which these functions were extended linearly. This amounts to sixteen inequalities. We consider only one of them, the requirement that the slope of $\hat{v}_{B}(0, u)$ be less than $\sqrt{2}$ on the interval $[x-\eta, \hat{x}]$. This is equivalent to

$$
\hat{v}_{B}(x-\eta, \hat{x})<\sqrt{2}(\hat{x}-(x-\eta)) .
$$

Both sides of this inequality are continuous functions of $\hat{x}$, and hence of $\hat{t}$. The inequality is true when $\hat{t}=t$, because in that case $\hat{x}=x$ and $\hat{v}_{B}$ has the same slope as $v_{B}$ in the interval. Therefore, this inequality holds if $\hat{t}$ is in a sufficiently small open neighborhood of $t$. The other fifteen inequalities impose similar constraints. So we need only to choose $\hat{t}$ sufficiently close to $t$ to make all of them hold, and then $\hat{v}_{A}, \hat{v}_{B}$, and $\hat{v}_{C}$ meet the requirements of the lemma.

\section{There is no finite protocol}

Theorem 1 There is no finite protocol that reliably finds an envy-free division of a cake.

Proof: We show that the theorem holds if the players' measures form a rigid measure system, even if the protocol is allowed to rely on that fact.

Suppose, on the contrary, that we have such a finite protocol. To reach a contradiction we will construct a rigid measure system with parameters $x, y, s$, and $t$, such that the protocol does not make a mark at $x$ or $y$ after any number of steps. Since the only envyfree allocations require cuts at $x$ and $y$, this means that the protocol cannot terminate in an envy-free division. This contradicts the assumption that the protocol is finite. The theorem follows.

We start with any rigid measure system $\left\{v_{i}\right\}$ with parameters $x, y, s$, and $t$, and make a sequence of alterations to it as follows. First, fix a value $\delta>0$ for which $1 / 3+\delta<t<$ $1 / 2-\delta$.

Begin the protocol with marks at 0 and 1.

Now let $N \geq 0$. Suppose that the protocol has proceeded for $N$ steps, and that no mark has been made at $x$ or $y$. Let $\eta>0$ be less than the smallest distance from $x$ or $y$ to any mark. Allow the protocol to continue through step $N+1$; suppose that the protocol selects player $i$ and real number $\alpha$ and asks player $i$ for its $\alpha$-point. If the resulting mark is not at $x$ or $y$, continue.

Suppose, instead, that the resulting mark is at $x$ or $y$. Then we need to alter the measures. From Lemma 3 , we can find a number $\hat{x} \neq x$, and a rigid measure system $\left\{\hat{v}_{i}\right\}$ with parameters $\hat{x}, \hat{y}, \hat{s}, \hat{t}$ such that 
- $v_{i}=\hat{v}_{i}$

- $v_{j}(u, v)=\hat{v}_{j}(u, v)$ for all $j$ and all existing marks $u, v$ (because all existing marks are at distance $\eta$ from $x$ and $y$ );

- $\hat{y} \neq y$; and

- $1 / 3+\delta<\hat{t}<1 / 2-\delta$.

Now we replace the system $\left\{v_{i}\right\}$ with the new system $\left\{\hat{v}_{i}\right\}$. The first two points above mean that all of the information available to player $i$, and to the protocol, is identical for both systems, so player $i$ still answers the $(N+1)$-st query with $x$ or $y$ - that is, not with the new parameters $\hat{x}$ or $\hat{y}$. Answers to previous queries are unchanged as well. So, for our new rigid measure system, the protocol has failed to find the right cuts through step $N+1$.

Continue in this way. It might be necessary to make infinitely many changes in the measures. But if we take the additional precaution of choosing the values of $\eta$ at each step so that they converge to zero, then only finitely many changes to the measures are made that affect any point. The successive choices of $\hat{t}$ are bounded away from $1 / 3$ and $1 / 2$, so that the measures actually converge to a rigid measure system. For that system, the protocol never makes a mark at the points where the cuts would have to be made.

\section{Cases with $n>3$}

Cases with $n>3$ can be handled in exactly the same way. When $n=5$, for example, a rigid measure system has parameters $x_{1}, x_{2}, x_{3}, x_{4}, s$, and $t$ satisfying $0<x_{1}<x_{2}<$ $x_{3}<x_{4}<1$ and $0<s<1 / 5<t<1 / 2$. The measures are governed by the table

\begin{tabular}{c|ccccc} 
& {$\left[0, x_{1}\right]$} & {$\left[x_{1}, x_{2}\right]$} & {$\left[x_{2}, x_{3}\right]$} & {$\left[x_{3}, x_{4}\right]$} & {$\left[x_{4}, 1\right]$} \\
\hline$v_{1}$ & $t$ & $t$ & $s$ & $s$ & $s$ \\
$v_{2}$ & $s$ & $t$ & $t$ & $s$ & $s$ \\
$v_{3}$ & $s$ & $s$ & $t$ & $t$ & $s$ \\
$v_{4}$ & $s$ & $s$ & $s$ & $t$ & $t$ \\
$v_{5}$ & $t$ & $s$ & $s$ & $s$ & $t$
\end{tabular}

As in the case of $n=3$, we arrange that there are exactly two envy-free allocations, and both of them require cuts at $x_{1}, x_{2}, x_{3}, x_{4}$. All steps of the proof are exactly the same as for $n=3$.

\section{Acknowledgments}

The author thanks the organizers of Dagstuhl Seminar 07261, and many of the participants, including especially Jiri Sgall, for helpful comments. 


\section{References}

[1] Barbanel, Julius B., and Steven J. Brams, "Cake division with minimal cuts: envyfree procedures for 3 persons, 4 persons, and beyond," Mathematical Social Sciences 48 (2004) 251-269.

[2] Brams, Steven J., and Alan D. Taylor, "An envy-free cake division protocol," American Mathematical Monthly 102 (1995) 9-18.

[3] Dagstuhl Seminar 07261, on Fair Division, June, 2007.

[4] Robertson, Jack, and William Webb, Cake-Cutting Algorithms: Be Fair If You Can, A. K. Peters, 1998 (ISBN 1568810768).

[5] Stromquist, Walter R., "How to Cut a Cake Fairly," American Mathematical Monthly 87 (1980) 640-644. See also addendum, 88 (1981) 613-614.

[6] Su, Francis Edward, "Rental Harmony: Sperner's Lemma in Fair Division," American Mathematical Monthly 106 (1999) 430-442.

[7] Woeginger, G. J., and Jiri Sgall, "On the complexity of cake cutting," Discrete Optimization 4 (2007) 213-220. 